\title{
Exploration of Word Learning From Multiple Perspectives
}

\author{
HARUMI KOBAYASHI \\ (TOKYo Denki UNIVERSITY)
}

\begin{abstract}
The phenomenon of children first producing words and then rapidly expanding their vocabulary is fascinating for both researchers and parents. At first glance, acquiring words and meanings may look relatively simple, but recent studies have revealed that word learning is actually a surprisingly complicated and difficult process. It is unlikely that only one approach can satisfactorily explore that process. The problem of word learning must be examined in many different ways from multiple perspectives. The present paper discusses studies of word learning from 5 different perspectives: (1) word learning biases and applications of those biases, (2) actions and pragmatic aspects of input, (3) developmental data and theoretical issues, (4) the fundamental ability to learn words, and (5) devices for word learning studies. The most important recent studies of word learning in Japan from the present author's viewpoint, mainly from international journals, are reviewed, but important domestic publications are also included and discussed.
\end{abstract}

Key Words : word learning, developmental issues, pragmatics, nouns and verbs, fundamental abilities

\section{Introduction}

The moment that children produce their first words are fascinating for both researchers and parents. For most children, this occurs at the beginning of their second year. But long before that, children show evidence that they understand the meaning of adults' utterances. After the production of their first words, children typically show rapid and more organized word learning after 18 months of age.

Initially, in the 1960's and 1970's, word learning research mainly studied the number of words or the size of children's vocabulary, but later, other aspects of word learning, such as the nature of the acquired words, operating principles or constraints in word learning, cognitive and physiological foundations of word leaning; use of symbols, bodily rhythms, gestures and actions; and the role of caretakers and other adults close to the child have been investigated. Haryu (1999), Ejiri (2001), and Saito (2003) published excellent reviews of language development, focusing upon recent studies in Japan, and issues of word learning have been discussed.

Studies of children's word learning have focused on relatively young children, such as toddlers and preschoolers, rather than on school children and ado- lescents. One reason for the emphasis on these relatively young children is that researchers are attracted to the rapid transition from the initial no-word stage to the abundant-word stage. Parents, teachers, and researchers are also interested in educational applications of these findings on language development in atypically developing preschool children.

Exploring the process and mechanism of word learning is important because associating meanings with words is essential for language development, and findings about the relevant processes and mechanisms may provide information for organizing and applying educational programs, and possibly for creating word learning machines. Because word learning is a surprisingly complicated and difficult process, it is unlikely that only one approach can satisfactorily explore it. The problem of word learning must be examined by many different researchers with many different approaches. If we come up with some conversions of the research results, we may consider ourselves lucky, but we must also welcome mixed or contrastive results, because such results may greatly enhance our knowledge of this field and stimulate new research.

The present paper discusses studies of word learn- 
ing from five different perspectives: (1) word learning biases and applications of those biases, (2) actions and pragmatic aspects of input, (3) developmental data and theoretical issues, (4) the fundamental ability for word learning, and (5) devices for word learning studies.

The most important recent studies in Japan of word learning, in the present author's view, have been selected for this review, mainly from international journals, but important domestic publications are also included and discussed. In addition, in order to benefit readers who are interested in Japanese research but are not competent in the Japanese language, articles written in English were given priority in the selection, but important ones in Japanese were also included.

Although most of the studies reviewed are pertinent to one of the five perspectives, studies may be related to more than one perspective.

\section{Word Learning Biases and Applications of Those Biases}

Young children learn words at a surprisingly rapid rate. One estimate of this rate is that a typical child learns nine new words per day after about one year and six months and before entering primary school (Carey, 1978). To explain this extremely rapid rate, Markman and her colleagues (Markman, 1989) proposed that young children use word-learning biases (assumptions, constraints) in order to relate word and their meanings rapidly.

The major assumptions include whole object assumption, taxonomic assumption, and mutual exclusivity. A whole object assumption is used if, for example, an object is present and a word is heard, and the child assumes that the word refers to the whole object, rather than a part of it. A taxonomic assumption is being used when an object is present and a word is heard, and the child assumes that the word refers to the category to which the object belongs. Mutual exclusivity means that the categories to which words are associated are mutually exclusive and do not overlap.

Some of the phenomena of young children's word learning can be explained by these assumptions. However, serious problems remain. For example, using word learning assumptions could potentially block children's learning some types of words. For instance, mutual exclusivity may block learning of words of different hierarchies, such as animal, dog, and hound, and a proper noun ("Blackie").

Thus, word learning biases or assumptions must be overridden when other available cues suggest that they should be. Recent studies have demonstrated that young children are sensitive to such cues and use cues such as eye-gaze direction, gestures, facial expressions, and actions, to associate words and meanings (Baldwin, 1991; Kobayashi, 1997, 1998; Tomasello \& Akhtar, 1995).

Other research has focused on the role of syntax in word learning, because in English syntax, there is a high correlation between syntactic classes and associated semantic categories. For example, counting nouns that are marked by the article " $a$ " or "an", and, in the plural, the suffix "-s", can be associated to objects; mass nouns can be associated to substances, and determiners can mark proper nouns and common nouns.

The interplay of syntactic classes and semantic categories in learning object words has been investigated (Gelman \& Taylor, 1984; Smith, Jones, \& Landau, 1992), but whether such an interplay of syntactic cues and semantic cues is a necessary part of word learning was not investigated in those studies.

Because, in Japanese, there are no grammatical markers flagging the distinctions between counting nouns and mass nouns, and between proper nouns and common nouns, whether children can learn words without the support of syntax can be studied in Japanese children. Imai and Haryu (2001) investigated how Japanese two- and four-year-olds assign meaning to novel nouns associated with familiar and unfamiliar animals and inanimate objects. Inspired by the experimental paradigm used by Gelman and Taylor (1984), Imai and Haryu (2001) showed children an unfamiliar stuffed animal and named it with a novel label, then presented four choices of subordinate, basic, superordinate, and unrelated categories, and asked the children whether each of the choices could be named by the novel label.

Their results showed that when a novel label was given to an unfamiliar object, children assumed that it was the name of the basic-level object category, 
regardless of whether the referent was an animate or an inanimate object. When the named object already had an established name, and if the object was an inanimate object, the children assumed the label to be a subordinate category label. But when the named object was an animal, they tended to interpret the label as a proper name. Imai and Haryu (2001) showed that Japanese two-year-olds can fast-map a noun to a meaning without syntactic cues.

When a new word is associated with a known category, young children may have several choices if they use a whole object assumption and mutual exclusivity. The children may simply reject the word, or may interpret the word to be the name of another category label such as a superordinate or subordinate label, if part names, proper names, and perceptual features are reasonably excluded.

Haryu and Imai (2002) examined how Japanese 3-year olds interpret a novel label given to a familiar artifact whose name is already known. In Study 1, the standard object was a familiar object that 3-yearolds can name (e.g., blue transparent plastic cup), and test objects were the standard object, a subordinate item (e.g., blue transparent plastic cup with a different color pattern), a basic level item (e.g., metal cup), a material item (e.g., a chunk of blue transparent plastic), and a distractor (e.g., a stuffed toy dog). Haryu and Imai (2002) asked the children whether each item could be named by a novel name. They found that the children chose a subordinate item.

In Study 2 (Haryu \& Imai, 2002), the standard object's shape was either typical (e.g., a typical spoon shape with a slight irregularity) or atypical (e.g., an atypical spoon with a square shape). They found that when the shape of the named object was typical, 3-year-olds mapped the new word to a subordinate category, whereas if the shape of the named object was atypical, 3-year-olds mapped the new word to a new category. Haryu and Imai (2002) concluded that children flexibly recruit cues from multiple sources, and that the cues are weighed in a hierarchical order, so that the children can determine the single most plausible solution.

Overextension refers to the phenomenon in which a child maps a category label (e.g., dog) to a category that is larger than adults' category (e.g., all four-legged animals, including cats, sheep, and horses). Markman (1989) suggested that an overextended category could become shaped closer to an adult's category as children learn new category labels. Because children assume that a newly learned label (e.g., horse) refers to a category that is mutually exclusive with an already learned category (e.g., four-legged animal), they may interpret the new label as a subordinate label or a new category label. Haryu and Imai's findings can be related to the Markman's position. One way of overriding overextension may be explained in terms of the results of Haryu and Imai (2002).

It is possible that caregivers provide useful information for learning word meanings of objects, but without any such input from caregivers, children still show a tendency to interpret words in certain ways. If word-learning biases can provide default assumptions, children might have a better start with this useful support for word learning.

\section{Actions and Pragmatic Aspects in Input}

A substantial amount of research has showed that young children can use actions and pragmatic cues such as eye gaze, facial expressions, and context in learning word meanings (Baldwin, 1991; Tomasello \& Akhtar, 1995). For example, in Tomasello and Akhtar's (1995) study, whether children learned a new word, "widget", for an action or an object depended upon what kind of preparatory behavior adults engaged in. Saylor, Baldwin, and Sabbagh (2002) used an adult's gestures of circling and ambiguous pointing at the whole object and the novel part, in order to examine children's inferences. They concluded that children's using pragmatic information provided by juxtaposition, that is, placing contrastive items side by side in space and/or time, can facilitate their learning of part names.

Kobayashi (1997) examined whether 2-year-olds attend to the relevant properties (shape or material) of solid objects in making inferences when adults demonstrate appropriate actions. The experimenter presented Japanese 2-year-olds a nonsense label with an unfamiliar target solid object that was either rigid (e.g., steel) or flexible (e.g., sponge), and then performed an action on the object that emphasized its shape (e.g., rolling) or its material (e.g., squeezing). The experimenter then asked the children, given the same label, 
to choose an object that matched the target in shape or in material. The results showed that the children used the action information to make their choices.

Based on her longitudinal observations of motherchild interactions with everyday objects such as a toothbrush and a ball, Kobayashi (2000) suggested that at least when children acquire names of tool-like objects, they first understand the relationship between their bodies and the objects, and what can be done with the objects in their own culture.

How young children learn part names can be an especially interesting problem from the perspective of actions and pragmatics. Because pointing is essentially ambiguous in specifying the speaker's referential intentions, the speaker is likely to add information such as by words, gestures, facial expressions, and eye gaze. Such information is important in learning any word, but in learning part names, the problem of referential ambiguity may become more salient. One reason is that parts and wholes are organized in a hierarchy in the environment. Any part of an object can include smaller parts, and a person must specify exactly what part in the environment is being referred to by others. Actions and pragmatic information seem to play a critical role in situations involving inferences about parts of objects.

The whole object assumption proposed by Markman and Wachtel (1988) is directly related to the issue of learning part names, although their approach focused on the word learning biases and principles discussed above. Markman and Wachtel (1988) showed that American three- and four-year-olds interpreted a novel name as the name of a whole object if an adult provided a part name (e.g., trachea) of an unfamiliar object (e.g., lung). In their research, the experimenter mentioned what the child was about to see by providing a label "trachea", and placed the picture of a lung on the table. Then she asked the child "Which one is the trachea? This whole thing [the experimenter circled the object (lung) with her index finger], or just this part [the experimenter pointed to the part (trachea)]?"

The children responded that the whole thing (lung) was the name of the referent. However, in the familiar object condition (e.g., fish), the children were able to choose a part (e.g., dorsal fin) when they were asked to point to a part as a referent of the novel name. Markman and Wachtel (1988) showed that children are able to learn part names when they know the names of the whole object, but whether children are able to use other cues to learn the names of object parts must still be examined.

Kobayashi (1998) investigated whether Japanese two-year-olds could learn novel part names of unfamiliar objects when an adult demonstrated actions upon parts of the objects. In the Kobayashi (1998) experiment, an experimenter pointed at, named, and acted upon a certain part (nut) of an unfamiliar object (a U-shaped bolt with a nut), or simply pointed at and named the part. Twenty-four Japanese two-yearolds were asked to choose a referent for the given name in forced-choice tests.

The children chose an isolated part (the nut) when the experimenter demonstrated an action upon the part. However, they chose a whole object of similar shape (a U-shaped bolt without a nut) when the experimenter did not demonstrate such an action. Kobayashi (1998) interpreted these results to mean that young children use pragmatic information provided by adults' actions upon object parts when they learn part names.

Kobayashi (2002) further demonstrated that even when the object part was not actually moved, 2-yearolds could make correct inferences as far as they observed gestures around the object's parts. In the Kobayashi (2002) experiment, the experimenter showed gestures related to the function of the part, such as turning the nut a few times around the bolt. The testing procedure was the same as in Kobayashi's (1998) study, and basically the same results were obtained. Thus, this research has demonstrated that young children are likely to be able to induce the referencing intentions of adults correctly by observing the adults' actions.

Recently, Kobayashi (2005) examined young children's interpretation of different types of pointing gestures in learning part names. As already mentioned, the meaning of pointing is ambiguous, because pointing itself only shows the direction that the pointer is paying attention to and normally does not specify an exact part of the environment that should be attended to. Kobayashi's (2005) study 
examined the effect of adults' pointing with touching parts of the object (touch-pointing) on learning novel part names of unfamiliar objects, in two-year-olds, four-year-olds, and adults. In Experiment 1, an experimenter touch-pointed at, named, and acted upon a certain part of an unfamiliar object, or simply touchpointed at and named the part, and asked 28 Japanese 4-year-olds to make inferences about novel part names. In Experiment 2, 30 2-year-olds, 31 4-yearolds, and 30 adults were tested in a similar experiment, except that the experimenter either touchpointed at the part or pointed from a distance of $7 \mathrm{~cm}$. The results indicated that the four-year-olds learned more part names when object parts were touchpointed, and that the four-year-olds' response patterns were similar to the adults' patterns. It was shown that adults' touch-pointing at object parts can be a useful pragmatic cue for learning part names.

Pragmatic aspects of input that are indicated by human bodily movements must be further investigated, and how the coordination of words and bodily movements provides useful information must be explored. I believe that such information can be directly applicable to designing educational programs and creating word-learning machines that make use of complicated and elaborate cues provided by the input.

\section{Developmental Data and Theoretical Issues}

Gentner (1982) claimed that children's early vocabulary may be predominantly nouns, because, in comparison with verbs, nouns refer to objects that have more distinctive contours and are more perceptually salient, and nouns map more concrete and cohesive concepts. This claim has been tested in various languages including Japanese, Korean, and Chinese, by many researchers, because to the extent that young children share basically the same perceptual and cognitive abilities, the noun advantage phenomenon must be universal, regardless of language typology. Language typology here refers to the distinction between a noun-privileged language and a verbprivileged language with respect to grammatical factors such as the null argument (e.g., a subject can be omitted in a sentence), relative complexity of verb morphology, and words that appear in the final position in the sentence.
Although a noun-biased vocabulary has been confirmed in English (Goldfield \& Reznick, 1990), young children showed a more balanced or even more verbbiased vocabulary in Chinese (Tardif, 1996) and Korean (Choi \& Gopnick, 1995). Japanese is considered to be a noun-privileged language, because of the null argument and the sentence final position of SOV, however, Japanese children seem to show a noun-dominant vocabulary (Ogura, 2000 ; Sakurai, 1999).

If language typology cannot explain these data, the riddle must be solved by some other factor. Fernald and Morikawa (1993) discussed cultural differences in interaction styles and views of child rearing in American and Japanese mothers. American mothers prefer object labeling whereas Japanese mothers prefer social routines. The nature of the mothers' input also varied, depending on the situation.

Miyata, Oshima-Takane, and Nishisawa (2004) analyzed longitudinal data collected over a span of ten months from four Japanese mother-child dyads. The dyads were given a set of toys (dolls, trains and rails, a picture book, a puzzle, and toy plates and food) and the mothers were instructed to interact with their children as they did in everyday life. Each session lasted 60 minutes; the mother-child interactions were videotaped.

Data from six different time points were transcribed in Japanese CHAT-format (Oshima-Takane \& MacWhinney, 1995). The children's speech was analyzed in terms of (1) word types and tokens, such as common nouns, full verbs, and verbal nouns, (2) noun/ verb ratio, (3) morphosyntactic measures such as mean length of utterance (MLU) and verb inflection variation index. Caregivers' speech was analyzed comparably, except that instead of the morphosyntactic measures, the percentage of verbs and nouns occurring in single-word utterances versus multi-word utterances was calculated, and analysis with speech act conducted.

The results showed that the child measures and maternal measures were significantly correlated, indicating that children whose speech was noun-biased had mothers whose speech was also noun-focused. If the mothers did not employ a noun-focused speech style, their children did not develop a noun bias. Miyata, Oshima-Takane, and Nishisawa (2004) con- 
cluded that the style of a mother's input, rather than general language typological factors, influences the composition of nouns and verbs of her child.

Recently, Imai, Haryu, and Okada (in press) approached this debate about noun-biased and verb-biased vocabulary in a novel experimental paradigm. They showed three- and five-year-olds and adults a series of videos in which a woman was doing a novel action using a novel object. For example, in the standard setting, a young woman was holding an object (a brown plastic drainpipe) in her right hand and pushing it outwards with a punching motion. Then, in the test situation, the child was shown two video clips of the woman doing the same action using a different object (a round metallic timer) or doing a different action with the same object (holding the object in her right hand, and tapping it against her left shoulder). While watching the standard event, a child heard either a novel noun ("X ga aru": "There is an $\mathrm{X}$ ") or a novel verb (“X-te'iru”: "X-ing").

The results showed that although five-year-olds and adults were successful in both conditions, threeyear-olds were successful only in the noun condition: they failed to extend verbs to the same action when the object in the action was changed. Imai et al. (in press) interpreted these results to mean that verb learning is more difficult for young children than noun learning.

In Studies 2 and 3, Imai et al. (in press) investigated why three-year-olds failed to make inferences about verb meaning. The researchers concluded that threeyear-olds were overly conservative, that is, they are unwilling to extend a novel verb to the same action when the object in the action is changed. Imai et al. (in press) emphasized that their results should not be interpreted to mean that Japanese children cannot learn verbs before they are five years old. Rather, they learn verbs as early as 18 months, but Imai et al. (in press) suggest that early verb learning is not complete and that it takes some time to learn the basic principles of verb generalization, that is, that verbs get generalized on the same action or relation while the objects are variables.

\section{Fundamental Ability to Learn Words}

Joint attention refers to two persons paying atten- tion to the same object. This was first explicitly investigated by Bruner and his colleagues (Scaife \& Bruner, 1975). The attended object can be any entity, including an everyday object, a person, or an event. Because joint attention is an essential part of human communication, it is estimated to be one of the most important achievements for human infants when acquiring language. Based on reviews of various perspectives and published experimental results, Oyabu (2004), in a cohesive and comprehensive book on joint attention, claimed that joint attention develops through five different stages: pre-joint attention (zen kyodochui), face-to-face joint attention (taimenteki kyodochui), joint attention with support from the caregiver (shijiteki kyodochui), joint attention with mutual intentions (ito kyoyuteki kyodochui), and joint attention with symbol sharing (symbol kyoyuteki kyodochui).

Pre-joint attention refers to newborn babies' inclination toward human faces, eyes, and voices. Adults are also sensitive to babies' signals. Pre-joint attention appears in an innately specified, primitive, and weak interaction of babies and caregivers. Face-toface joint attention appears between two months and six months of age. In this stage of joint attention, the baby is obviously looking at other's eyes, and socially important smiles occur.

Joint attention with caregiver's support appears from six months of age. Joint attention can be established in two ways: A caregiver may detect the direction of the infant's eye gaze and turn to gaze at the object, or an infant may detect the direction of the caregiver's eye gaze and turns to gaze at the object.

Oyabu (2004) pointed out that at this stage, the caregiver's role seems more important for establishing joint attention: the frequency of the caregiver turning the eyes toward the object is higher at this stage. Joint attention with mutual intentions refers to an infant's behavior of actively detecting and controlling the direction of an adult's gaze. Joint attention with mutual intentions appears at 9 to 12 months of age. The infant follows the other's eye gaze, consults the other's face (social referencing), and imitates the other's behavior.

Joint attention with symbol sharing appears between 15 and 18 months. At this stage, the child can share the meaning of the other's intentions, and 
consequently can acquire the meaning of symbols and words. Because the object of joint attention can be both concrete and abstract objects, the child starts to use symbols to refer to absent referents, such as past and future events.

Some studies have focused in detail on some of these developmental stages, in order to investigate exactly what kind of mechanisms operate to establish some type of joint attention. In the face-to-face joint attention that appears between two months and six months, infants are considered to interact with objects or other persons only dyadically. At around 9 to 12 months of age, a new set of behavior emerges which can be considered to be triadic interaction, in the sense that it involves a coordination of the child's interactions with both objects and adults, resulting in a referential triangle of child, adult, and the object of which they share attention (Tomasello, 1999). Nadel and Tremblay-Leveau (1999) recognized that a personperson-person (P-P.P) system can be observed among infants younger than six months, and suggested that this system may be a precursor of the person-objectperson (P-O-P) system.

Amano, Kezuka, and Yamamoto (2004) proposed another type of triadic interaction system between P-P.P and P-O-P: person-person(object)-person (P-P(O)$P$ ). They pointed out that this type of interaction can be observed when an adult holds an object in his/her hand $[\mathrm{P}(\mathrm{O})$ refers to this situation], and the infant looks at and/or reaches for it.

The following episode is an example of the P$\mathrm{P}(\mathrm{O})$ - $\mathrm{P}$ system. During a meal, a spoon on the table does not attract the infant's attention. An infant may look only at the adult's face. Then the adult picks up the spoon and brings food by the spoon to her mouth, and the infant's attention may shift from the adult's face to the spoon. Now the infant wants to hold the spoon and eats with it.

Amano et al.'s (2004) interest was to specify a precursor of joint attention established in a system of P-O-P, and investigate the shifting of attention from an adults' face to an adult's hand in three- and fourmonth-olds. The infants were presented different types of hand gestures including pointing and head inclinations while their mothers and a stranger pointed to objects. The results were as follows: (1) after encountering an averted head repeatedly, the infants shifted their attention from the adult's face to the moving hand and objects, thus they oriented to what the adult was attending to. (2) The moving head increased the rate of infants' turning their head in the same direction as the adult's. Amano et al. (2004) concluded that an averted head and eyes play an important role in infants' orienting to an adult's hand, and the infant looked at the hand as the adult manipulated an object. They suggested that infants' orientation to the adult's hand is a precursor stage of joint attention in the P-O-P system.

\section{Devices for Word Learning Studies}

The Japanese MacArthur Communicative Development Inventories (JCDIs).

It is very important to evaluate the levels of language and communication development of young children properly for better consultation and intervention at hospitals, preschools, and institutions of health care and day care. Language delay is one of the most frequent concerns that caregivers and teachers have. Although there is a need for tools to evaluate children's language and communication skills correctly, unfortunately, until recently, there has been no standardized way to evaluate children's language development properly.

The Japanese MacArthur Communicative Development Inventories (JCDIs), published in 2004, can be a useful tool for such an evaluation. The Japanese MacArthur Communicative Development Inventories, and the two technical manuals that accompany these inventories, were published by the Kyoto International Social Welfare Exchange Center (Ogura \& Watamaki, 2004; Watamaki \& Ogura, 2004).

The Japanese MacArthur Communicative Development Inventories include two parts: Words and Gestures, which is suitable for children aged 8 to 18 months, and Words and Grammar, which is suitable for children aged 16 to 36 months. Words and Gestures investigates productive or comprehensive vocabularies of 711 words and 5 items for the use of words. Words and Grammar investigates 100 items, including the longest sentence that the child utters.

The Japanese MacArthur Communicative Development Inventories were developed based on care- 
givers' responses about their children's use of gestures, words, and sentences. They were standardized on Japanese children, based on the MacArthur Communicative Development Inventories (MCDIs) originally developed by Fenson, Dale, Reznick, Thal, Bates, Hartung, Pethick, and Reilly (1993) and used to investigate children from the stage of preverbal communication to the stage of grammatical constructions. Ogura and Watamaki (Ogura \& Watamaki, 2004; Watamaki \& Ogura, 2004) collected data from more than 2000 Japanese caregivers, and standardized the inventories using data from observations of interactions of children and caregivers in university playrooms.

The Japanese Child Language Data Exchange System (JCHAT).

It is important to collect and analyze data from children's utterances in order to study language development. In 1984, twenty researchers in the U.S.A. and Europe initiated a collaboration in which data from children's utterances collected by individual researchers would be shared through the Child Language Data Exchange System (CHILDES), which initially was directed by MacWhinney and Snow. The development of computer technology contributed greatly to the development of this system. CHAT, a transcript system of utterances, and CLAN, a software program for analysis including mean length of utterance (MLU), analysis of morphemes, and searching for specific words and sentences, were developed, and databases of various languages were added.

In 1992, a group of Japanese researchers established the JCHAT (Japanese CHAT) project to make a transcript system for the Japanese language. At present, transcribed data from more than twenty-eight languages are included in the system. Today, the TalkBank project, supported by the National Science Foundation, makes it possible to see video clips of a child and a caretaker or teacher on the internet.

A guidebook, Introduction to the CHILDES Utterances Database (Hatsuwa Database CHILDES Nyumon), edited by Miyata (2004), was published. This is a handy and understandable guidebook with many attractive illustrations and an excellent guide for beginners and intermediate users.

The Bibliography Database for Language Acquisition Research (JBIB).
Some time before 2000, a group of researchers interested in language development started working to establish a Japanese database for reference when studying language development. The Bibliography Database for Language Acquisition Research (JBIB) was established and has been directed by Ono at Aichi Shukutoku University and other researchers (Ono, Otomo, Kobayashi, Shirai, Shirai, Sugiura, Hirakawa, Hirakawa, Yukawa, \& Wakabayashi, 2001). This database includes academic publications such as books, journal articles, proceedings, technical reports, dissertations, and other materials in the areas of first language acquisition, second language acquisition, bilingualism, foreign language education, and atypical language development. The project started in 2000, with the support of the Ministry of Education (now the Ministry of Education, Culture, Sports, Science and Technology), and a Bibliography Database for Language Acquisition Research website was constructed and made accessible in 2004.

Using JBIB Search (http://cow.lang.nagoya-u.ac.jp/ jbib/jbibsearch.html) is simple: the website can be instantly accessed without any constraints; the author name(s) and/or key word(s) from the title of articles are required for a database search. People who use the system to find information about articles are only required to mention the JBIB URL and some referential information in the references of their articles. Unfortunately, updating the database does not seem to be perfect, but it is a useful source of information for those who are searching for articles.

\section{Conclusions}

It is unlikely that only one approach can satisfactorily explore the process of word learning. Rather, it must be examined from multiple perspectives and in many different ways. The present paper discussed studies of word learning conducted recently in Japan from five different perspectives. Future research with these and additional perspectives must explore these fascinating phenomena more fully in order to enhance our knowledge about and understanding of word learning.

\section{References}

Amano, S., Kezuka, E., \& Yamamoto, A. 2004 
Infant shifting attention from an adult's face to an adult's hand: A precursor of joint attention. Infant Behavior and Development, 27, 64-80.

Baldwin, D. A. 1991 Infants' contribution to the achievement of joint reference. Child Development, 62, 875-890.

Carey, S. 1978 The child as a word learner. In M. Halle, G. Miller, \& J. Bresnan (Eds.), Linguistic theory and psychological reality. Cambridge, MA: MIT Press.

Choi, S., \& Gopnik, A. 1995 Early acquisition of verbs in Korean: A cross-linguistic study. Journal of Child Language, 22, 497-529.

Ejiri, K. 2001 Language development from the viewpoint of the relation between language and body. Progress of Child Psychology, 40, 77-100. (in Japanese)

Fenson, L., Dale, P. S., Reznick, J. S., Thal, D., Bates, E., Hartung, J., Pethick, S., \& Reilly, J. 1993 MacArthur Communicative Development Inventories: User's guide and technical manual. San Diego, CA: Singular Publishing Group.

Fernald, A., \& Morikawa, H. 1993 Common themes and cultural variations in Japanese and American mothers' speech to infants. Child Development, 64, 637-656.

Gelman, S. A., \& Taylor, M. 1984 How two-yearold children interpret proper and common names for unfamiliar objects. Child Development, 55, 1535-1540.

Gentner, D. 1982 Why nouns are learned before verbs: Linguistic relativity versus natural partition. ing. In S. A. Kuczaj (Ed.), Language development: vol. 2. Language, thought, and culture. Hillsdale, NJ: Lawrence Erlbaum Associates. Pp. 301-334.

Goldfield, B., \& Reznick, S. 1990 Early lexical acquisition: Rate, content, and the vocabulary spurt. Journal of Child Language, 17, 171-193.

Haryu, E. 1999 Language development. Progress of Child Psychology, 38, 81-109. (in Japanese)

Haryu, E., \& Imai, M. 2002 Reorganizing the lexicon by learning a new word: Japanese children's inference of the meaning of a new word for a familiar artifact. Child Development, 73, 1378-1391.

Imai, M., \& Haryu, E. 2001 Learning proper nouns and common nouns without clues from syntax.
Child Development, 72, 787-802.

Imai, M., Haryu, E., \& Okada, H. (in press) Mapping novel nouns and verbs onto dynamic action events: Are verb meanings easier to learn than noun meanings for Japanese children? Child Development.

Kobayashi, H. 1997 The role of actions in making inferences about the shape and material of solid objects among Japanese 2-year-old children. Cognition, 63, 251-269.

Kobayashi, H. 1998 How two-year-old children learn novel part names of unfamiliar objects. Cognition, 68, B41-B51.

Kobayashi, H. 2000 Actions can direct word learning: Interrelations between affordances and object words. Studies in Language Sciences, 1, 61-72.

Kobayashi, H. 2002 Learning novel part names by observing adults' gestures. Studies in Language Sciences, 2, 149-156.

Kobayashi, H. 2005 Strategies that mothers use to teach children part names of everyday objects. Manuscript submitted for publication.

Markman, E. M. 1989 Categorization in children: Problems of induction. Cambridge, MA: MIT Press, Bradford Books.

Markman, E. M., \& Wachtel, G. F. 1988 Children's use of mutual exclusivity to constrain the meanings of words. Cognitive Psychology, 20, 121-157.

Miyata, S. 2004 An introduction to utterance data base CHILDES. Tokyo: Hitsuji Shobo. (in Japanese)

Miyata, S., Oshima-Takane, Y., \& Nishisawa, H. 2004 Noun bias in early Japanese vocabulary and characteristics of maternal speech. Studies in Language Sciences, 3, 87-101.

Nadel, J., \& Tremblay-Leveau, H. 1999 Early interpersonal timing and the perception of social contingencies. In P. Rochat (Ed.), Early social cognition: Understanding others in the first months of life. London: Lawrence Erlbaum Associates. Pp. 189-212. Ogura, T. 2000 Standardization of the Japanese MacArthur Communicative Development Inventories. Tokyo: Kenkyu Seika Hokokusho. (in Japanese)

Ogura, T., \& Watamaki, T. 2004 Technical manual for the Japanese MacArthur Communicative Develop- 
ment Inventory: Words and gestures. Kyoto: Kyoto International Social Welfare Exchange Center.

Ono, K., Otomo, K., Kobayashi, H., Shirai, H., Shirai J., Sugiura, M., Hirakawa, M., Hirakawa, Y., Yukawa, E., \& Wakabayashi, S. 2001 JBIB search: http://cow.lang.nagoyau.ac.jp/jbib/jbibsearch.html

Oshima-Takane, Y., \& MacWhinney, B. 1995 CHILDES manual for Japanese. Montreal: McGill University.

Oyabu, Y. 2004 Joint Attention: The developmental process from birth to 2 years 6 months. Tokyo: Kawashima Shoten. (in Japanese)

Saito, K. 2003 Child-centered studies of language development. Progress of Child Psychology, 42, 253-285. (in Japanese)

Sakurai, C. 1999, August Explaining a Japanese child's noun bias: A pragmatic analysis. In Early vocabulary in Japanese children: An appraisal of current facts and factors. Symposium conducted at the meeting of the 12th World Congress of Applied Linguistics, AILA '99, Tokyo. Symposium Handout, pp. 11-13.

Saylor, M. M., Baldwin, D. A., \& Sabbagh, M. A. 2002 Children use whole-part juxtaposition as a pragmatic cue to word meaning. Developmental Psychology, 38, 993-1003.

Scaife, M., \& Bruner, J. S. 1975 The capacity for joint attention in the infant. Nature, 253, 265-266.

Smith, L B., Jones, S. S., \& Landau, B. 1992 Count nouns, adjectives, and perceptual properties in children's novel word interpretations. Developmental Psychology, 28, 273-286.

Tardif, T. 1996 Nouns are not always learned before verbs: Evidence from Mandarin speakers' early vocabulary. Developmental Psychology, 32, 492-504.

Tomasello, M. 1999 The cultural origin of human cognition. Cambridge, MA: Harvard University Press.

Tomasello, M., \& Akhtar, N. 1995 Two-year-olds use pragmatic cues to differentiate reference to objects and actions. Cognitive Development, 10, 201-224.

Watamaki, T., \& Ogura T. 2004 Technical manual for the Japanese MacArthur Communicative Development Inventory: Words and grammar. Kyoto: Kyoto International Social Welfare Exchange Center. 